\title{
Observations across nearby spiral bulges
}

\author{
A. E. Sansom ${ }^{1}$, S. I. Loubser ${ }^{1}$, P. Sánchez-Blázquez ${ }^{1}$ and \\ I. K. Söchting ${ }^{2}$ \\ ${ }^{1}$ Centre for Astrophysics, University of Central Lancashire, Preston, Lancs, UK \\ email: AESansom@uclan.ac.uk \\ ${ }^{2}$ Astrophysics, Oxford University, Denys Wilkinson Building, Keeble Road, Oxford, UK \\ email: iks@astro.ox.ac.uk
}

\begin{abstract}
The bulges of spiral galaxies may be old, as in our Galaxy, or may possess younger stars, as evidenced in spectroscopic line strengths in some external bulges. Bulges look similar to elliptical galaxies, but their formation history is expected to differ due to the presence of the disc and different formation mechanisms. This project extends the numbers of high signal-to-noise, broad coverage spectra to a larger sample of bulges in order to test conflicting ideas about their age distributions. New Gemini long-slit observations will be used to derive stellar population ages and histories across 30 bulges. Here we present preliminary results from the sample.
\end{abstract}

Keywords. galaxies: bulges, galaxies: formation, galaxies: stellar content

\section{Stellar Populations in Bulges}

Controversy over the ages of bulges arises because of age/metallicity degeneracy and mass/age degeneracy of a younger component. Colours were thought to reveal old ages in bulges (Peletier et al. 1999). Spectroscopic observations of 11 bulges observed with the WHT revealed younger luminosity weighted average ages (1.5 to $6 \mathrm{Gyr})$ and lower $[\alpha / \mathrm{Fe}]$ abundance ratios than in bright ellipticals (Proctor \& Sansom 2002, their figure 12). This was confirmed using these same data, but fitting different simple stellar population models, by Thomas \& Davies (2006). There is a weak trend for bulge ages to increase with velocity dispersion. This small sample and others were insufficient to clearly define any trends with Hubble type for bulges. For this we need a larger sample.

Bulges may form by more than one route. Some probably form via mergers, whilst others could be formed by internal dynamics, such as the effects of bars. If such secular evolution is the main mechanism then discs should influence bulges. This was not seen by Thomas \& Davies (2006) who showed that bulges and ellipticals had similar line strengths at a given velocity dispersion. This was also found from a sample of low luminosity ellipticals by Northeast (2006). Higher signal-to-noise spectra going further out in radius are needed to test these models, to distinguish between central and global population characteristics in bulges (Peletier et al. 2007).

\section{Galaxy Sample and Observations}

We selected a statistically significant sample of nearby spiral galaxies that are nearly edge-on, so that the spectrograph slit could be placed across the bulge minor axis. Galaxies of type Sa to Sbc were included. AGN were avoided as was the Galactic plane. We obtained long-slit spectroscopic observations of 30 galaxies with GEMINI North and South telescopes in 2006B and 2007A observing semester. Our aim is to measure the spread of star formation histories amongst the different Hubble types of bulges and spatially 

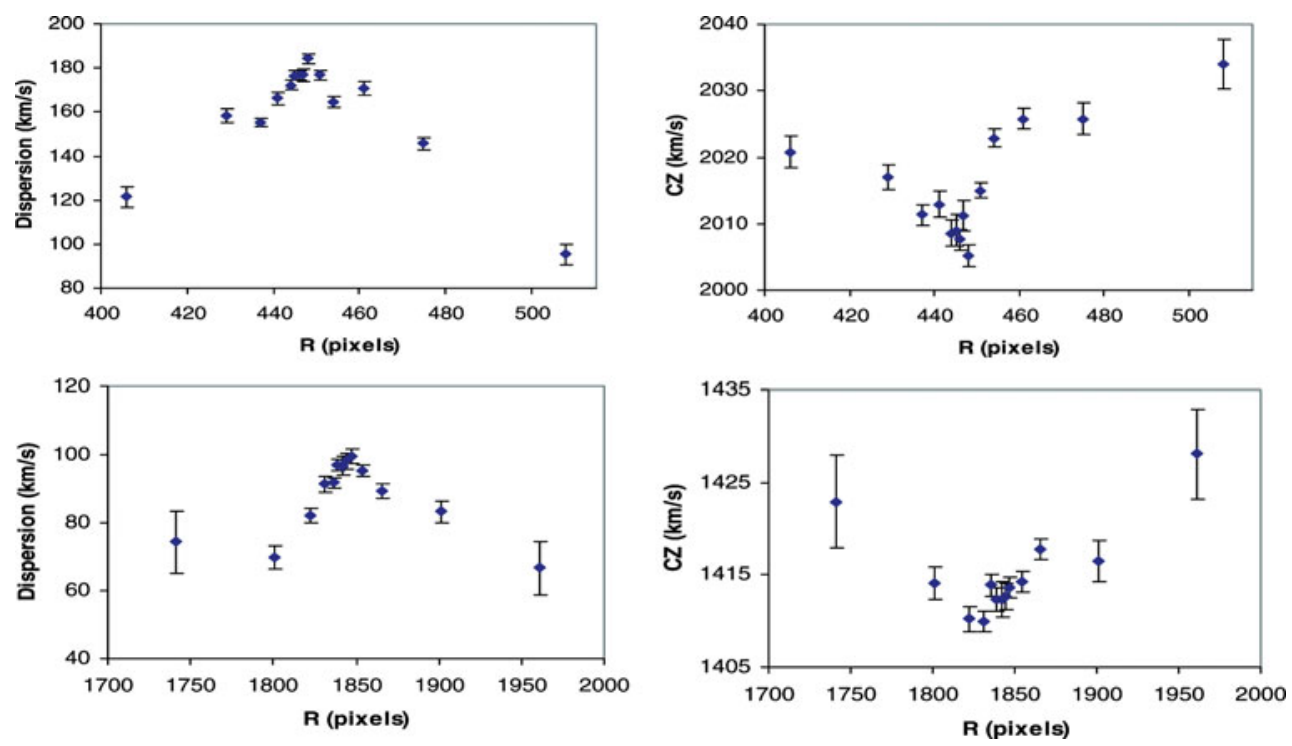

Figure 1. Two examples of spatially resolved kinematics of Gemini bulges data, showing decreasing velocity dispersion with radius in NGC2648 (upper) and NGC779 (lower). Velocity profiles are also shown. 1 pixel $=0.146 "$.

across bulges. To calibrate these data we make use of observations of Lick standard stars available in the Gemini Science Archive from previous programmes (PI: B. Miller).

The data are currently being measured for spatially resolved velocities and velocity dispersions across our sample of bulges, using the reduceme package (figure 1). Kinematics are needed for accurate calibration of spectral line-strengths. They are also useful for revealing subcomponents in the bulges. Centrally peaked dispersion profiles are seen in the two galaxies shown, and low levels of rotation are observed, due to the need to align the slit so as to obtain sufficiently bright guide stars with GMOS. Errors are obtained from Monte-Carlo simulations of the data, given an error array that incorporates Poisson noise due to the original data and sky subtraction.

With spectroscopic optical absorption line-strengths covering a broad spectral range, we will measure average luminosity-weighted galaxy ages and metallicities to within $\sim 20 \%$. The results will be compared with published data from related galaxy types to help us classify the behaviour of stellar populations in different types of spiral bulges. Spatially resolved ages and metallicity determinations as a function of radius across the bulges will help to disentangle different formation scenarios.

\section{References}

Northeast, M. S. 2006, PhD thesis, University of Central Lancashire

Peletier, R. et al. 1999, MNRAS, 310, 703

Peletier, R. et al. 2007, MNRAS, accepted, astro-ph/07042839

Proctor, R. N. \& Sansom, A. E. 2002, MNRAS, 333, 517

Thomas, D. \& Davies R. L. 2006, MNRAS, 366, 510 